\title{
Simultaneidade de comportamentos de risco para a obesidade em adultos das capitais do Brasil
}

\author{
Simultaneity of risk behaviors for obesity in adults in the capitals \\ of Brazil
}

Anne Ribeiro Streb (http://orcid.org/0000-0001-9195-4210) ${ }^{1}$

Giovani Firpo Del Duca (https://orcid.org/0000-0003-0893-2032) ${ }^{1}$

Robert Passos da Silva (https://orcid.org/0000-0002-9456-1997) ${ }^{1}$

Jucemar Benedet (https://orcid.org/0000-0002-2058-6040) ${ }^{1}$

Deborah Carvalho Malta (https://orcid.org/0000-0002-8214-5734) ${ }^{2}$

${ }^{1}$ Centro de Desportos, Universidade Federal de Santa Catarina. R. Eng. Agronômico Andrei

Cristian Ferreira s/n, Trindade. 88040-900

Florianópolis SC Brasil. anne.streb@posgrad.ufsc.br

${ }^{2}$ Departamento de Enfermagem Materno Infantil e Saúde Pública, Escola de Enfermagem, Universidade Federal de Minas Gerais. Belo Horizonte MG Brasil.

\begin{abstract}
The scope of this study was to verify the association between risk behaviors and obesity in adults (18 to 59 years of age) in Brazilian capitals. It involved a cross-sectional population-based study conducted by telephone interview. The self-reported variables were obesity, defined by weight and height (BMI $\left.\geq 30 \mathrm{~kg} / \mathrm{m}^{2}\right)$ and risk behaviors: physical inactivity ( $\leq 149$ minutes/week), excess sedentarism ( $\geq 4$ hours/day), frequent consumption of sweets ( $\geq 5$ days/week) and meat with fat and/or chicken with skin ( $\geq 1$ day/week). The simultaneity of risk behaviors was analyzed for reasons of observed and expected prevalence. The Poisson and multinomial logistic regressions were used in crude analyses and adjusted for sociodemographic factors. Among the 35,448 adults, the simultaneous consumption of meat with fat, excess sedentarism and physical inactivity (PR: 1.94, 95\% CI: 1.45, 2.60), as well as the presence of the four risk behaviors (PR: 1.72, 95\% CI: 1.16, 2.53) were associated with obesity in men. In women, the simultaneous consumption of sweets and meat with fat (PR: 1.77, 95\% CI: 1.19, 2.66) was also associated with the outcome. In both sexes, there was an increase in the tendency of obesity according to the sum total of risk behaviors.
\end{abstract}

Key words Risk factors, Obesity, Cross-sectional studies
Resumo Objetivou-se verificar a associação da simultaneidade de comportamentos de risco com a obesidade em adultos (18 a 59 anos) das capitais do Brasil. Trata-se de um estudo transversal de base populacional, realizado por entrevista telefônica. As variáveis autorrelatadas foram a obesidade, definida pelo peso e altura (IMC $\geq 30$ $\mathrm{kg} / \mathrm{m}^{2}$ ) e os comportamentos de risco: inatividade fisica ( $\leq 149 \mathrm{~min} / \mathrm{sem})$, tempo sentado em excesso ( $\geq 4 \mathrm{~h} / \mathrm{dia})$, consumo frequente de doces $(\geq 5$ dias/sem) e de carnes vermelhas com gordura e/ou frango com pele ( $\geq 1$ dia/sem). A simultaneidade dos comportamentos de risco foi analisada por razões das prevalências observadas e esperadas. Foram utilizadas as regressões de Poisson e logistica multinomial, em análises por sexo, brutas e ajustadas por fatores sociodemográficos. Dentre os 35.448 adultos, nos homens, a simultaneidade do consumo de carnes com gordura, tempo sentado em excesso e inatividade física (RP: 1,94; IC95\%: $1,45 ; 2,60)$, assim como a presença dos quatro comportamentos de risco (RP: 1,72; IC95\%: 1,16; 2,53), associaram-se à obesidade. Em mulheres, o consumo simultâneo de doces e de carnes com gordura (RP: 1,77; IC95\%: 1,19; 2,66) também se associou ao desfecho. Em ambos os sexos, observouse aumento da tendência de obesidade conforme o somatório de comportamentos de risco.

Palavras-chave Fatores de risco, Obesidade, Estudos transversais 


\section{Introdução}

A obesidade tem despertado preocupações constantes no âmbito da saúde pública, principalmente porque é evidente sua associação com outras doenças crônicas não transmissíveis (DCNTs) e mortalidade ${ }^{1}$. Na população adulta, a obesidade está fortemente correlacionada a diversas enfermidades, tais como síndrome metabólica, incapacidade funcional, doenças cardiovasculares, acometimentos osteomiarticulares ${ }^{2}$ e, ainda, transtornos mentais, como a depressão $0^{3}$. Este cenário também implica em uma demanda financeira custosa para a saúde pública ${ }^{4,5}$.

A causalidade da obesidade permeia aspectos genéticos e relacionados ao estilo de vida, sendo difícil atribuir uma proporção exata para cada um destes fatores, no entanto, está bem estabelecido na literatura que o desencadeamento da obesidade tem forte relação com os hábitos de vida $^{6}$. Neste contexto, estudos que investigaram os processos de ganho, perda e manutenção da massa corporal têm adotado como principal foco os fatores de risco comportamentais modificáveis, principalmente a atividade física ${ }^{7,8} \mathrm{e}$ o comportamento alimentar' 9 . Tem sido observado que a diminuição dos níveis de atividade física e a adoção de uma dieta rica em gorduras estão positivamente associados à obesidade ${ }^{10-12}$. O mesmo não se pode afirmar com relação ao consumo de doces. Os resultados dos estudos conduzidos acerca da temática são divergentes, principalmente aqueles desenvolvidos com adultos ${ }^{13,14}$.

Além disso, muitas pesquisas têm sido desenvolvidas na perspectiva de investigar tais comportamentos de modo isolado e, é necessário considerar, que estes comportamentos são complexos e se inter-relacionam. Ao analisar de forma simultânea indicadores de atividade física e hábitos alimentares, é possível observar se a exposição para um determinado comportamento está relacionada à adoção de outros ${ }^{15}$. Entender como os comportamentos de risco se relacionam entre si e se há diferença entre eles em homens e mulheres se torna pertinente para uma melhor compreensão da ocorrência de obesidade, assim como para a organização de ações estratégicas preventivas em saúde pública. Nesse sentido, o objetivo do presente estudo foi verificar a associação entre a simultaneidade de comportamentos de risco e a obesidade em homens e mulheres adultos residentes nas capitais do Brasil.

\section{Métodos}

Este estudo transversal, de caráter descritivo e de base populacional, analisa microdados do Sistema de Vigilância de Fatores de Risco e Proteção para Doenças Crônicas por Inquérito Telefônico (VIGITEL) realizado de fevereiro a dezembro de 2015. Este vem sendo implantado desde $2006 \mathrm{em}$ todas capitais do Brasil e no Distrito Federal e tem auxiliado no monitoramento da frequência, distribuição e evolução dos principais determinantes das DCNT's. A população alvo do presente estudo foi somente de adultos de 18 a 59 anos de ambos os sexos, moradores das 27 capitais brasileiras e que possuíam em sua residência uma linha de telefone fixo.

Para a realização da amostragem probabilística, houve a seleção das linhas telefônicas com a utilização de um sorteio sistemático de cinco mil linhas, estratificadas por código de endereçamento postal. Estas foram divididas em subamostras de 200 linhas cada, reproduzindo a mesma proporção de linhas por região da cidade ou prefixo telefônico. Estipularam-se duas mil entrevistas como número mínimo a ser realizado em cada capital. Após a identificação das linhas elegíveis, sorteou-se os participantes para a realização da entrevista, dentre todos os adultos residentes no domicílio contatado. As estimativas produzidas foram ajustadas à população adulta brasileira residente nas capitais cobertas pela rede de telefonia fixa, com o uso do peso pós-estratificação considerando o sexo, a faixa etária e o nível de escolaridade, ajustando-se de forma a representar a população, a partir da ponderação de dados. O peso pós-estratificação de cada indivíduo da amostra foi calculado pelo método rake. O detalhamento do plano amostral, os passos da amostragem e a aplicação de pesos estão disponíveis em sua totalidade no relatório do VIGI$\mathrm{TEL}^{16}$. Todos os dados foram obtidos por meio de ligação telefônica em forma de entrevista guiada através de um questionário previamente valida$\mathrm{do}^{17}$, preenchido com recursos simultâneos do computador.

O índice de massa corporal (IMC) foi utilizado como desfecho e calculado a partir do autorrelato do peso e da altura de cada indivíduo por meio das seguintes questões: " $\mathrm{O}(\mathrm{a}) \mathrm{sr}(\mathrm{a})$ sabe seu peso (mesmo que seja valor aproximado)?" e "O(a) sr(a) sabe sua altura?". Para a classificação do IMC utilizou-se os pontos de corte para o diagnóstico de obesidade $\left(\geq 30 \mathrm{~kg} / \mathrm{m}^{2}\right)$. Para suprir as perdas amostrais, fez-se a imputação de dados por meio do tratamento estatístico hot 
deck, que levou em consideração as variáveis sociodemográficas de idade, sexo, escolaridade e raça/cor ${ }^{16}$.

Os comportamentos de risco para a obesidade investigados como exposições no estudo foram: o consumo muito frequente de doces (consumo de doces em $\geq 5$ dias/semana $)^{18}$, consumo habitual de carnes vermelhas com gordura e/ou frango com pele (relato de ingesta de carne com gordura e/ou frango com pele $)^{19}$, o elevado tempo sentado diário ( $\geq 4$ horas/dia) e a inatividade física (não cumprimento das recomendações de prática semanal de 150 minutos/semana considerando todos os domínios $)^{20}$.

A estatística descritiva contou com o uso de frequências absolutas (n) e relativas (\%). A análise de simultaniedade se deu por meio da formação das 16 possíveis combinações dos quatro comportamentos de risco (consumo muito frequente de doces, consumo habitual de carnes vermelhas com gordura e/ou frango com pele, tempo sentado diário e inatividade física). Estas combinações foram examinadas quanto à sua prevalência observada $(\mathrm{O})$ e esperada $(\mathrm{E})$, bem como a respectiva relação $\mathrm{O} / \mathrm{E}$. A prevalência esperada foi calculada com base na multiplicação das probabilidades individuais do status (presença ou ausência) de cada comportamento estudado. Para testar a associação destas 16 combinações com a obesidade utilizou-se a regressão de Poisson bruta e ajustada extratificada por sexo. $\mathrm{Na}$ análise ajustada, foram incluídas as variáveis idade e escolaridade, em decorrência da possibilidade de confundimento e/ou modificação de efeito. Foi empregado também a regressão logística multinomial para verificar se o somatório de dois ou mais comportamentos de risco estavam associados com a obesidade. Em todas as análises inferenciais foram considerados estatisticamente significantes os valores de $\mathrm{p} \leq 0,05$, sendo que para a associação do somatório de comportamentos utilizou-se o teste de tendência para determinar o valor $\mathrm{p}$. Os resultados foram expressos como razões de prevalência (RP), razão de ODDS (OR) e intervalos de confiança de 95\% (IC 95\%). Os dados foram analisados pelo software Stata, versão 13.0, levando em consideração os fatores de ponderação da amostragem complexa. O procedimento de consentimento livre e esclarecido foi realizado no momento da entrevista. A aprovação do projeto VIGITEL foi obtida pelo Comitê Nacional de Ética em Pesquisa do Ministério da Saúde, no ano de 2013.

\section{Resultados}

De um total de 76.703 linhas elegíveis, ao final, foram completadas 54.174 entrevistas, sendo que destes, $83,1 \%$ eram adultos (idade entre 18 e 59 anos), o que representa 35.448 pessoas. A Tabela 1 traz a descrição das características sociodemográficas, dos comportamentos de risco e o percentual de obesidade nos adultos das capitais brasileiras de acordo com o sexo. Destaca-se que, dentre os comportamentos de risco investigados, a prevalência mais elevada nas mulheres foi de inatividade física $(41,0 \%)$ e nos homens foi o consumo habitual de carnes vermelhas com gordura e/ou frango com pele $(55,5 \%)$.

As Tabelas 2 e 3 apresentam os resultados da interação entre os quatro comportamentos de risco e sua associação com a obesidade em homens e mulheres adultos das capitais brasileiras, respectivamente. A combinação mais frequente nos homens foi a ausência dos quatro comportamentos de risco $(16,6 \%)$, já para as mulheres foi a presença isolada de inatividade física $(18,5 \%)$. Dentro dos agrupamentos menos frequentes, para os homens deu-se o agrupamento 8 (presença de consumo muito frequente de doces e inatividade física) em 1,0\%, enquanto a ausência isolada de inatividade física foi para mulheres em 1,4\%. Para ambos os sexos, a prevalência esperada do agrupamento 11 (presença simultânea de tempo sentado e inatividade física) era muito inferior da que foi encontrada (1,8 para $7,0 \mathrm{em}$ homens e 4,6 para 10,3 em mulheres) sinalizando um quadro de sedentarismo nesta população.

Os resultados da razão entre a prevalência observada e a esperada permite verificar a força de agrupamento destes comportamentos, o que estabelece a probabilidade de que eles aconteçam simultaneamente. Nesse sentido, o agrupamento 11 para homens e 10 para mulheres mostraramse mais provável de ocorrer em simultaneidade (O/E: 4,85 IC95\%: 4,57; 4,73; O/E: 5,14 IC95\%: 4,$16 ; 4,73$, respectivamente). Isso significa dizer que a inatividade física e o tempo sentado para homens agrupados aos comportamentos alimentares positivos têm uma probabilidade $485 \%$ mais alta de acontecer do que se esses comportamentos fossem totalmente independentes.

Dentre os 35.448 adultos, nos homens, a simultaneidade do consumo de carnes com gordura, tempo sentado em excesso e inatividade física (RP: 1,94 ; IC95\%: 1,45; 2,60), assim como a presença dos quatro comportamentos de risco (RP: 1,72; IC95\%: 1,16; 2,53) associaram-se à obesidade. Em mulheres, o consumo simultâneo de 
Tabela 1. Características sociodemográficas e de saúde dos adultos das capitais brasileiras de acordo com o sexo $(\mathrm{n}=35.448)(2015)$.

\begin{tabular}{|c|c|c|c|c|c|c|}
\hline \multirow[t]{2}{*}{ Variáveis } & \multicolumn{3}{|c|}{$\begin{array}{c}\text { Feminino } \\
(\mathrm{n}=21.069)\end{array}$} & \multicolumn{3}{|c|}{$\begin{array}{l}\text { Masculino } \\
(\mathrm{n}=14.379)\end{array}$} \\
\hline & $\mathbf{n}$ & $\%^{\mathrm{a}}$ & IC95\% & n & $\%^{\mathrm{a}}$ & IC95\% \\
\hline \multicolumn{7}{|l|}{ Escolaridade (anos) } \\
\hline 0 a 08 & 3.723 & 27,3 & 26,$0 ; 28,7$ & 2.600 & 29,8 & 28,$0 ; 31,6$ \\
\hline 09 a 11 & 8.377 & 41,6 & 40,$2 ; 42,9$ & 6.118 & 42,6 & 41,$0 ; 44,2$ \\
\hline$\geq 12$ & 8.969 & 31,1 & 29,$8 ; 32,2$ & 5.661 & 27,6 & 26,$2 ; 28,9$ \\
\hline \multicolumn{7}{|c|}{ Consumo muito frequente de doces } \\
\hline Sim & 4.089 & 23,9 & 22,$8 ; 25,2$ & 2.369 & 18,3 & 17,$1 ; 19,7$ \\
\hline Não & 16.980 & 76,1 & 74,$8 ; 77,3$ & 12.010 & 81,7 & 80,$3 ; 82,9$ \\
\hline \multicolumn{7}{|c|}{$\begin{array}{l}\text { Consumo habitual de carnes vermelhas com gordura e/ou } \\
\text { frango com pele }\end{array}$} \\
\hline $\operatorname{Sim}$ & 4.373 & 23,2 & 22,$0 ; 24,4$ & 5.834 & 55,5 & 53,$8 ; 57,2$ \\
\hline Não & 16.696 & 76,8 & 75,$5 ; 77,9$ & 8.545 & 44,5 & 42,$7 ; 46,1$ \\
\hline \multicolumn{7}{|c|}{ Tempo sentado em excesso } \\
\hline $\operatorname{Sim}$ & 8.800 & 40,9 & 39,$5 ; 42,2$ & 7.261 & 47,2 & 45,$5 ; 48,8$ \\
\hline Não & 12.269 & 59,1 & 57,$7 ; 60,4$ & 7.118 & 52,8 & 51,$1 ; 54,4$ \\
\hline \multicolumn{7}{|l|}{ Inatividade física } \\
\hline Sim & 10.718 & 41,0 & 39,$5 ; 42,2$ & 5.217 & 33,5 & 31,$9 ; 35,1$ \\
\hline Não & 10.351 & 59,0 & 57,$7 ; 60,4$ & 9.162 & 66,4 & 64,$8 ; 68,0$ \\
\hline \multicolumn{7}{|l|}{ Obesidade } \\
\hline Sim & 3.727 & 19.1 & 17,$8 ; 20,1$ & 2.735 & 18,3 & 17,$0 ; 19,6$ \\
\hline Não & 17.342 & 80,9 & 79,$8 ; 82,1$ & 11.644 & 81,7 & 80,$3 ; 82,9$ \\
\hline
\end{tabular}

doces e de carnes com gordura (RP: 1,77; IC95\%: $1,19 ; 2,66)$ também se associou ao desfecho.

A Tabela 4 apresenta o somatório de 2 ou mais comportamentos não saudáveis e sua associação com a obesidade, em comparação àqueles que apresentam um ou nenhum comportamento de risco. Nota-se tendência de aumento de chances de obesidade a medida que se acumulam comportamentos de ricos $(\mathrm{p}=<0,001)$. Nos homens, observou-se uma associação positiva com gradiente linear.

\section{Discussão}

O objetivo principal deste estudo foi investigar os agrupamentos de comportamentos de risco à saúde e sua associação com a obesidade de acordo com o sexo. Destaca-se como principal achado, para os homens, a associação do agrupamento dos quatro comportamentos de risco estudados com a obesidade. Enquanto isso, para as mulheres, a adoção de comportamentos saudáveis parece ser mais determinante para a não ocorrên- cia de tal doença. Além disso, nossos resultados apontam que, conforme há acúmulo de comportamentos de risco, incrementa-se a probabilidade de ocorrência da obesidade. Esta tendência já foi reportada em um inquérito nacional na população americana adulta, onde a combinação de diversos comportamentos de risco como fumar, ingerir bebidas alcóolicas em excesso, ter baixos níveis de atividade física e comportamento dietético inadequado foi preditora de obesidade ${ }^{21}$. De fato, adultos que desenvolvem sobrepeso ou obesidade tendem a possuir baixos níveis de atividade física, tempo elevado de exposição à televisão e distúrbios relacionado ao sono, indicando que esses comportamentos parecem se agrupar e expor os indivíduos a um risco aumentado ${ }^{22}$.

É importante destacar a alta prevalência de inatividade física e de tempo sentado detectados na população estudada. O agrupamento que possui a inatividade física como único comportamento de risco englobou $18,5 \%$ das mulheres avaliadas, quando se esperava uma prevalência de somente $4,4 \%$ nesta população. Esta informação se torna preocupante, pois a exposição 
Tabela 2. Simultaneidade de fatores de risco para obesidade em homens das capitais brasileiras. Porcentagem observada, esperada e a razão de prevalência para obesidade com intervalo de confiança de $95 \%$ ( $\mathrm{n}=14.379)$ (2015).

\begin{tabular}{|c|c|c|c|c|c|c|c|c|c|c|c|}
\hline & & $\begin{array}{l}\text { resen } \\
\text { rres d }\end{array}$ & e rise & & & & & & & Bruta & Ajustada \\
\hline & CD & CG & TS & IF & $\begin{array}{c}\% \text { de } \\
\text { obesidade }\end{array}$ & $\mathbf{n}$ & $\begin{array}{c}\% \\
\text { Observada }^{a} \\
\end{array}$ & $\begin{array}{c}\% \\
\text { Esperada }^{\mathrm{a}}\end{array}$ & $\begin{array}{c}\text { O/E } \\
(\mathrm{IC} 95 \%)\end{array}$ & $\begin{array}{c}\text { RP(IC } \\
95 \%)\end{array}$ & $\begin{array}{c}\text { RP(IC } \\
95 \%)\end{array}$ \\
\hline 1 & - & - & - & - & 16,1 & 2262 & 16,6 & 15,7 & $1,07(1,02 ; 1,11)$ & 1 & 1 \\
\hline 2 & + & - & - & - & 21,0 & 282 & 2,4 & 8,9 & $0,23(0,20 ; 0,26)$ & $\begin{array}{r}1,30 \\
(0,74 ; 2,29)\end{array}$ & $\begin{array}{r}1,33 \\
(0,75 ; 2,35)\end{array}$ \\
\hline 3 & - & + & - & - & 17,2 & 1601 & 14,6 & 16,0 & $0,74(0,70 ; 0,78)$ & $\begin{array}{r}1,06 \\
(0,79 ; 1,44)\end{array}$ & $\begin{array}{r}1,08 \\
(0,81 ; 1,47)\end{array}$ \\
\hline 4 & - & - & + & - & 17,5 & 1998 & 13,4 & 10,7 & $1,39(1,32 ; 1,44)$ & $\begin{array}{r}1,08 \\
(0,81 ; 1,45)\end{array}$ & $\begin{array}{r}1,25 \\
(0,93 ; 1,70)\end{array}$ \\
\hline 5 & - & - & - & + & 18,3 & 1112 & 7,3 & 3,1 & $2,67(2,51 ; 2,83)$ & $\begin{array}{r}1,13 \\
(0,83 ; 1,56)\end{array}$ & $\begin{array}{r}1,00 \\
(0,74 ; 1,38)\end{array}$ \\
\hline 6 & + & + & - & - & 17,2 & 303 & 3,1 & 6,1 & $0,37(0,32 ; 0,41)$ & $\begin{array}{r}1,06 \\
(0,67 ; 1,70)\end{array}$ & $\begin{array}{r}1,14 \\
(0,72 ; 1,83)\end{array}$ \\
\hline 7 & + & - & + & - & 9,7 & 414 & 3,0 & 10,9 & $0,28(0,25 ; 0,31)$ & $\begin{array}{r}0,60 \\
(0,36 ; 1,00)\end{array}$ & $\begin{array}{r}0,75 \\
(0,45 ; 1,25)\end{array}$ \\
\hline 8 & + & - & - & + & 11,9 & 142 & 1,0 & 3,2 & $0,33(0,28 ; 0,39)$ & $\begin{array}{r}0,74 \\
(0,40 ; 1,37)\end{array}$ & $\begin{array}{r}0,76 \\
(0,42 ; 1,40)\end{array}$ \\
\hline 9 & - & + & + & - & 15,3 & 1357 & 10,5 & 9,1 & $1,11(1,04 ; 1,16)$ & $\begin{array}{r}0,95 \\
(0,70 ; 1,29)\end{array}$ & $\begin{array}{r}1,15 \\
(0,84 ; 1,56)\end{array}$ \\
\hline 10 & - & + & - & + & 18,8 & 796 & 6,4 & 2,1 & $2,80(2,61 ; 3,00)$ & $\begin{array}{r}1,16 \\
(0,82 ; 1,66)\end{array}$ & $\begin{array}{r}1,07 \\
(0,75 ; 1,51)\end{array}$ \\
\hline 11 & - & - & + & + & 21,9 & 1150 & 7,0 & 1,8 & $4,85(4,57 ; 5,14)$ & $\begin{array}{r}1,35 \\
(1,01 ; 1,84)^{\star}\end{array}$ & $\begin{array}{r}1,36 \\
(1,01 ; 1,84)\end{array}$ \\
\hline 12 & + & + & + & - & 20,9 & 391 & 3,4 & 6,2 & $0,47(0,42 ; 0,52)$ & $\begin{array}{r}1,29 \\
(0,72 ; 2,30)\end{array}$ & $\begin{array}{r}1,64 \\
(0,92 ; 2,91)\end{array}$ \\
\hline 13 & + & + & - & + & 21,7 & 130 & 1,2 & 2,2 & $0,45(0,37 ; 0,53)$ & $\begin{array}{r}1,34 \\
(0,72 ; 2,51)\end{array}$ & $\begin{array}{r}1,28 \\
(0,69 ; 2,36)\end{array}$ \\
\hline 14 & + & - & + & + & 25,7 & 283 & 2,0 & 1,2 & $1,75(1,55 ; 1,96)$ & $\begin{array}{r}1,07 \\
(0,68 ; 1,70)\end{array}$ & $\begin{array}{r}1,25 \\
(0,79 ; 1,96)\end{array}$ \\
\hline 15 & - & + & + & + & 28,5 & 965 & 5,9 & 1,8 & $3,99(3,74 ; 4,25)$ & $\begin{array}{r}1,77 \\
(1,31 ; 2,38)^{*}\end{array}$ & $\begin{array}{r}1,94 \\
(1,45 ; 2,60)^{\star}\end{array}$ \\
\hline 16 & + & + & + & + & 24,9 & 291 & 2,2 & 1,2 & $1,76(1.57 ; 1,98)$ & $\begin{array}{r}1,54 \\
(1,04 ; 2,32)^{*}\end{array}$ & $\begin{array}{r}1,72 \\
(1,16 ; 2,53)^{\star}\end{array}$ \\
\hline
\end{tabular}

CD: consumo muito frequente de doces. CG: consumo habitual de carnes vermelhas com gordura e/ou frango com pele. TS: tempo sentado em excesso. IF: inatividade física. +: presença de fator de risco. - : ausência de fator de risco. n: frequência absoluta de cada agrupamento; O/E: \% observada /\% esperada; RP: razão de prevalência; IC95\%; ${ }^{a}$ Valores ponderados. Análises ajustadas para idade e escolaridade.

demasiada a estes comportamentos implica no agravamento dos seus efeitos nocivos ${ }^{23}$, tem forte associação com mortalidade por todas as causas, doenças cardiovasculares, diabetes tipo 2 e síndrome metabólica ${ }^{24}$. Além disso, a redução dos níveis de atividade física nesse grupo, em especial, já vem sendo relatada ${ }^{25}$. No entanto, mesmo com a recorrência da elevada prevalência de inatividade física em diferentes populações ${ }^{26,27}$, a literatura tem reportado carência em estratégias adequadas para o monitoramento do comportamento se- dentário, bem como, a necessidade de intervenções que despertem estilos de vida ativo ${ }^{23}$.

Neste estudo, a capacidade de agrupamento do consumo muito frequente de doces e os demais comportamentos não foi claramente identificada em ambos os sexos. No entanto, a hipótese que vem sendo levantada por alguns autores ${ }^{28,29}$, é de que o consumo de snackes, doces e industrializados está associado ao tempo de tela, caracterizado como uma das principais formas de comportamento sedentário. Em um recente estudo de 
Tabela 3. Simultaneidade de fatores de risco para obesidade em mulheres das capitais brasileiras. Porcentagem observada, esperada e a razão de prevalência para obesidade com intervalo de confiança de 95\% ( $\mathrm{n}=21.069)$ (2015).

\begin{tabular}{|c|c|c|c|c|c|c|c|c|c|c|c|}
\hline & \multicolumn{4}{|c|}{$\begin{array}{c}\text { Presença de } \\
\text { fatores de risco }\end{array}$} & \multirow[b]{2}{*}{$\begin{array}{c}\% \text { de } \\
\text { obesidade }\end{array}$} & \multirow[b]{2}{*}{$\mathbf{n}$} & \multirow[b]{2}{*}{$\begin{array}{c}\% \\
\text { Observada }^{a} \\
\end{array}$} & \multirow[b]{2}{*}{$\begin{array}{c}\% \\
\text { Esperada }^{a}\end{array}$} & \multirow[b]{2}{*}{$\begin{array}{c}\mathrm{O} / \mathrm{E} \\
(\mathrm{IC} 95 \%)\end{array}$} & \multirow{2}{*}{$\begin{array}{c}\text { Bruta } \\
\text { RP(IC } \\
95 \%)\end{array}$} & \multirow{2}{*}{$\begin{array}{c}\text { Ajustada } \\
\text { RP(IC } \\
95 \%) \\
\end{array}$} \\
\hline & $\mathrm{CD}$ & CG & TS & IF & & & & & & & \\
\hline 1 & - & - & - & - & 21,4 & 3629 & 17,9 & 18,3 & $\begin{array}{r}1,06 \\
(1,02 ; 1,09)\end{array}$ & 1 & 1 \\
\hline 2 & + & - & - & - & 11,1 & 574 & 3,8 & 18,9 & $\begin{array}{r}0,16 \\
(0,14 ; 0,17)\end{array}$ & $\begin{array}{r}0,51 \\
(0,31 ; 0,85)^{\star}\end{array}$ & $\begin{array}{r}0,55 \\
(0,34 ; 0,90)^{*}\end{array}$ \\
\hline 3 & - & + & - & - & 19,3 & 896 & 4,7 & 13,1 & $\begin{array}{r}0,36 \\
(0,34 ; 0,38)\end{array}$ & $\begin{array}{r}0,90 \\
(0,69 ; 1,18)\end{array}$ & $\begin{array}{r}0,94 \\
(0,71 ; 1,23)\end{array}$ \\
\hline 4 & - & - & + & - & 12,1 & 2373 & 10,8 & 4,8 & $\begin{array}{r}2,64(2,53 ; \\
2,75)\end{array}$ & $\begin{array}{r}0,57 \\
(0,44 ; 0,73)^{\star}\end{array}$ & $\begin{array}{r}0,74 \\
(0,58 ; 0,96)^{*}\end{array}$ \\
\hline 5 & - & - & - & + & 23,6 & 3641 & 18,5 & 4,4 & $\begin{array}{r}4,41 \\
(4,26 ; 4,55)\end{array}$ & $\begin{array}{r}1,10 \\
(0,90 ; 1,34)\end{array}$ & $\begin{array}{r}1,03 \\
(0,85 ; 1,26)\end{array}$ \\
\hline 6 & + & + & - & - & 35,4 & 222 & 2,0 & 5,0 & $\begin{array}{r}0,24 \\
(0,20 ; 0,27)\end{array}$ & $\begin{array}{r}1,65 \\
(1,08 ; 2,52)^{\star}\end{array}$ & $\begin{array}{r}1,77 \\
(1,19 ; 2,66)^{\star}\end{array}$ \\
\hline 7 & + & - & + & - & 6,9 & 629 & 3,6 & 3,4 & $\begin{array}{r}0,98 \\
(0,90 ; 1,05)\end{array}$ & $\begin{array}{r}0,32 \\
(0,17 ; 0,59)^{*}\end{array}$ & $\begin{array}{r}0,44 \\
(0,24 ; 0,80)^{\star}\end{array}$ \\
\hline 8 & + & - & - & + & 18,4 & 604 & 4,0 & 3,2 & $\begin{array}{r}1,02 \\
(0,94 ; 1,10)\end{array}$ & $\begin{array}{r}0,86 \\
(0,57 ; 1,29)\end{array}$ & $\begin{array}{r}0,93 \\
(0,61 ; 1,40)\end{array}$ \\
\hline 9 & - & + & + & - & 18,5 & 637 & 3,4 & 13,6 & $\begin{array}{r}0,25 \\
(0,23 ; 0,27)\end{array}$ & $\begin{array}{r}0,87 \\
(0,57 ; 1,29)\end{array}$ & $\begin{array}{r}1,17 \\
(0,79 ; 1,75)\end{array}$ \\
\hline 10 & - & + & - & + & 26,9 & 961 & 5,7 & 1,2 & $\begin{array}{r}4,44 \\
(4,16 ; 4,73)\end{array}$ & $\begin{array}{r}1,26 \\
(0,97 ; 1,63)\end{array}$ & $\begin{array}{r}1,24 \\
(0,95 ; 1,61)\end{array}$ \\
\hline 11 & - & - & + & + & 16,5 & 2159 & 10,3 & 4,6 & $\begin{array}{r}2,52 \\
(2,41 ; 2,63)\end{array}$ & $\begin{array}{r}0,77 \\
(0,60 ; 1,00)\end{array}$ & $\begin{array}{r}0,95 \\
(0,74 ; 1,21)\end{array}$ \\
\hline 12 & + & + & + & - & 7,6 & 246 & 1,4 & 3,6 & $\begin{array}{r}0,37 \\
(0,32 ; 0,42)\end{array}$ & $\begin{array}{r}0,36 \\
(0,15 ; 0,83)^{\star}\end{array}$ & $\begin{array}{r}0,53 \\
(0,23 ; 1,22)\end{array}$ \\
\hline 13 & + & + & - & + & 19,7 & 265 & 1,8 & 0,8 & $\begin{array}{r}1,71 \\
(1,50 ; 1,92)\end{array}$ & $\begin{array}{r}0,92 \\
(0,59 ; 1,45)\end{array}$ & $\begin{array}{r}0,97 \\
(0,63 ; 1,48)\end{array}$ \\
\hline 14 & + & - & + & + & 14,1 & 788 & 5,1 & 1,2 & $\begin{array}{r}3,52 \\
(3,27 ; 3,77)\end{array}$ & $\begin{array}{r}0,66 \\
(0,45 ; 0,96)^{\star}\end{array}$ & $\begin{array}{r}0,91 \\
(0,62 ; 1,34)\end{array}$ \\
\hline 15 & - & + & + & + & 15,3 & 780 & 4,4 & 3,3 & $\begin{array}{r}1,27 \\
(1,18 ; 1,36)\end{array}$ & $\begin{array}{r}0,72 \\
(0,53 ; 0,98)^{\star}\end{array}$ & $\begin{array}{r}0,90 \\
(0,65 ; 1,24)\end{array}$ \\
\hline 16 & + & + & + & + & 18,5 & 366 & 2,6 & 0,9 & $\begin{array}{r}2,28 \\
(2,05 ; 2,52) \\
\end{array}$ & $\begin{array}{r}0,88 \\
(0,54 ; 1,38) \\
\end{array}$ & $\begin{array}{r}1,18 \\
(0,75 ; 1,88) \\
\end{array}$ \\
\hline
\end{tabular}

CD: consumo muito frequente de doces. CG: consumo habitual de carnes vermelhas com gordura e/ou frango com pele. TS: tempo sentado em excesso. IF: inatividade física. +: presença de fator de risco. - : ausência de fator de risco. n: frequência absoluta de cada agrupamento; O/E: \% observada /\% esperada; RP: razão de prevalência; IC95\%; ${ }^{a}$ Valores ponderados. Análises ajustadas para idade e escolaridade.

base populacional realizado em Estocolmo, capital da Suécia, foi verificado que pessoas com perfis de estilo de vida desfavoráveis em relação ao consumo de álcool, doces e inatividade física, são mais propensas a permanecerem sedentárias ${ }^{30}$. Ainda, o mesmo estudo sugere que intervenções combinadas devam ser feitas para que haja uma tentativa de mudança destes comportamentos.

É interessante observar também os resultados que apontaram o consumo de doces em excesso e ter comportamento sedentário ( $\geq 4 \mathrm{~h} /$ dia) como proteção para a obesidade em mulheres (agrupamentos 4 e 7). Na literatura atual, é possível encontrar estudos nos quais o consumo de doces não foi diretamente associado com a obesidade $^{31,32}$, bem como, o comportamento sedentário, que é hegemônico na rotina das pessoas nos dias atuais ${ }^{24,33}$. Acredita-se que a prática de atividade física regular pode agir como mecanismo compensatório destes comportamentos, pois 
Tabela 4. Associação entre o somatório de comportamentos de risco e obesidade em adultos das capitais brasileiras ( $\mathrm{n}=34.448)$ (2015).

\begin{tabular}{|c|c|c|c|c|c|c|c|c|}
\hline & \multicolumn{4}{|c|}{$\begin{array}{l}\text { Masculino } \\
(\mathrm{n}=14.379)\end{array}$} & \multicolumn{4}{|c|}{$\begin{array}{l}\text { Feminino } \\
(\mathrm{n}=21.069)\end{array}$} \\
\hline & \multicolumn{2}{|c|}{ Bruta } & \multicolumn{2}{|c|}{ Ajustada } & \multicolumn{2}{|c|}{ Bruta } & \multicolumn{2}{|c|}{ Ajustada } \\
\hline & $\begin{array}{c}\text { OR } \\
(\mathrm{IC} 95 \%) \\
\end{array}$ & $\begin{array}{c}\mathrm{p} \\
\text { valor }\end{array}$ & $\begin{array}{c}\text { OR } \\
(\mathrm{IC} 95 \%) \\
\end{array}$ & $\begin{array}{c}\mathrm{p} \\
\text { valor }\end{array}$ & $\begin{array}{c}\text { OR } \\
(\mathrm{IC} 95 \%) \\
\end{array}$ & $\begin{array}{c}\mathrm{p} \\
\text { valor }\end{array}$ & $\begin{array}{c}\text { OR } \\
(\mathrm{IC} 95 \%) \\
\end{array}$ & $\begin{array}{c}\mathbf{p} \\
\text { valor }\end{array}$ \\
\hline 0 ou 1 & 1 & $<0,001$ & 1 & $<0,001$ & 1 & 0,06 & 1 & $<0,001$ \\
\hline 2 & $\begin{array}{r}0,99 \\
(0,80 ; 1,21)\end{array}$ & & $\begin{array}{r}1,02 \\
(0,83 ; 1,26)\end{array}$ & & $\begin{array}{r}0,98 \\
(0,81 ; 1,17)\end{array}$ & & $\begin{array}{r}1,16 \\
(0,96 ; 1,40)\end{array}$ & \\
\hline 3 & $\begin{array}{r}1,51 \\
(1,14 ; 2,01)\end{array}$ & & $\begin{array}{r}1,66 \\
(1,24 ; 2,22)\end{array}$ & & $\begin{array}{r}0,71 \\
(0,55 ; 0,90)\end{array}$ & & $\begin{array}{r}0,97 \\
(0,75 ; 1,25)\end{array}$ & \\
\hline 4 & $\begin{array}{r}1,59 \\
(0,98 ; 2,59) \\
\end{array}$ & & $\begin{array}{r}1,73 \\
(1,08 ; 2,77) \\
\end{array}$ & & $\begin{array}{r}0,94 \\
(0,55 ; 1,62) \\
\end{array}$ & & $\begin{array}{r}1,39 \\
(0,81 ; 2,36) \\
\end{array}$ & \\
\hline
\end{tabular}

OR = razão de ODDS. IC95\% = intervalo de confiança de 95\%.

Análises ajustadas para idade e escolaridade.

parece estar mais associada com a obesidade ${ }^{34,35}$, se mostrando presente na combinação referida. Ainda assim, é razoável considerar que a população estudada é de adultos e que as implicações negativas destas atitudes adotadas ao longo dos anos, refletir-se-ão predominantemente quando idosos, progredindo para quadros de multimorbidade de doenças crônicas, incapacidade funcional e mortalidade precoce.

Este estudo apresentou como limitações a coleta das variáveis por autorrelato, o que pode ter ocasionado viés de aferição, especialmente nas variáveis peso, altura e consumo alimentar, bem como a possibilidade de causalidade rever$\mathrm{sa}$, inerente a delineamentos transversais. Outro ponto que merece destaque é o fato de o Sistema VIGITEL utilizar em sua metodologia de seleção amostral, o sorteio de linhas de telefone fixo. Tal fato requer cautela e permite que a extrapolação dos achados seja feita exclusivamente para a população residente nas capitais do país. Embora a quantidade da cobertura telefônica fixa venha decaindo nos últimos anos, o fato de o estudo realizar a ponderação dos dados com base no perfil sociodemográfico da população brasileira, ameniza o possível viés de seleção. Outros trabalhos já foram conduzidos nessa perspectiva de comparabilidade dos achados de pesquisas com amostragem domiciliar e telefônica ${ }^{36}$. Todavia, pontos positivos como o tamanho amostral e o panorama epidemiológico de estudos como este, bem como a análise da simultaneidade de distintos comportamentos de risco à saúde merecem destaque, uma vez que grande parte das pesquisas tendem a trata-los de forma isolada, como se fossem totalmente independentes.

Sendo assim, conclui-se que os comportamentos de risco relacionados à obesidade foram distintos conforme o sexo. Em homens, a obesidade esteve associada de forma positiva com o agregamento dos comportamentos de risco investigados. Em especial, as combinações que incluíram o tempo sentado e a inatividade física foram aquelas mais fortemente associadas à obesidade. Já em mulheres, a obesidade se associou de forma negativa com o agregamento dos comportamentos saudáveis. Considerando as evidências apresentadas e o cenário difícil no que concerne à adoção de hábitos saudáveis na população adulta, sugere-se que futuras intervenções levem em consideração os comportamentos relacionados ao estilo de vida de forma interligada, preferencialmente com uma abordagem multidisciplinar e de acordo com o sexo. 


\section{Colaboradores}

AR Streb e GF Del Duca participaram de todas as etapas do estudo. RP Silva, J Benedet e DC Malta contribuíram com a redação do artigo e a análise dos resultados. Todos os autores aprovaram a versão final a ser publicada e se declaram responsáveis pelo manuscrito na garantia da exatidão e integridade de qualquer parte da obra.

\section{Referências}

1. Kearns K, Dee A, Fitzgerald AP, Doherty E, Perry IJ. Chronic disease burden associated with overweight and obesity in Ireland: the effects of a small BMI reduction at population level. BMC Public Health 2014; 14(1):143-150

2. Williams EP, Mesidor M, Winters K, Dubbert PM, Wyatt SB. Overweight and Obesity: Prevalence, Consequences, and Causes of a Growing Public Health Problem. Curr Obes Rep 2015; 4(3):363-370.

3. Preiss K, Brennan L, Clarke D. A systematic review of variables associated with the relationship between obesity and depression. Obes Rev 2013; 14(11):906918.

4. Bahia, L, Araújo, DV. Impacto econômico da obesidade no Brasil. Rev Hosp Univer Pedro Ernesto 2014; 13(1):13-17.

5. Biener A, Cawley J, Meyerhoefer C. The High and Rising Costs of Obesity to the US Health Care System. $J$ Gen Intern Med 2017; 32 (Supl. 1):6-8.

6. Mendonça CP, Anjos LA. Aspectos das práticas alimentares e da atividade física como determinantes do crescimento do sobrepeso/obesidade no Brasil Dietary and physical activity factors as determinants of the increase. Cad Saude Publica 2004; 20(3):698-709.

7. O’Reilly GA, Cook L, Spruijt-Metz D, Black DS. Mindfulness-based interventions for obesity-related eating behaviours: a literature review. Obes Rev 2014; 15(6):453-461.

8. Swift DL, Johannsen NM, Lavie CJ, Earnest CP, Church TS. The Role of Exercise and Physical Activity in Weight Loss and Maintenance. Prog Cardiovasc Dis 2014; 56(4):441-447.

9. Khan I, Ahmad S. Influence of Life Style, Nutrition and Obesity on Immune Response: A Global Issue. $J$ Food Process Technol 2017; 8(1):2-9.

10. Chastin SFM, Palarea-Albaladejo J, Dontje ML, Skelton DA. Combined Effects of Time Spent in Physical Activity, Sedentary Behaviors and Sleep on Obesity and Cardio-Metabolic Health Markers: A Novel Compositional Data Analysis Approach. Plos One 2015; 10(10):1-19.

11. Kahn SE, Hull RL, Utzschneider KM. Mechanisms linking obesity to insulin resistance and type 2 diabetes. Nature 2006; 444(7121):840-846.

12. Murillo R, Albrecht SS, Daviglus ML, Kershaw KN. The role of physical activity and sedentary behaviors in explaining the association between acculturation and obesity among Mexican-American adults. Am J Health Promot 2015; 30(1):50-57.

13. Stanhope KL. Sugar consumption, metabolic disease and obesity: The state of the controversy. Crit Rev Clin Lab Sci 2016; 53(1):52-67.

14. Gaino NM, da Silva MV. Consumo de frutose e impacto na saúde humana. Segur Aliment E Nutr 2011; 18(2):88-98.

15. Glanz K, Rimer BK, Viswanath K. Health behavior and health education: theory, research, and practice. $4^{\mathrm{a}} \mathrm{ed}$. San Francisco: John Wiley \& Sons; 2008. 
16. Brasil. Ministério da Saúde (MS). Vigitel Brasil 2015 vigilância de fatores de risco e proteção para doenças crônicas por inquérito telefônico: estimativas sobre frequência e distribuição sociodemográfica de fatores de risco e proteção para doenças crônicas nas capitais dos 26 estados brasileiros e no Distrito Federal em 2015. Brasília: MS; 2016.

17. Monteiro CA, Moura EC, Jaime PC, Lucca A, Florindo AA, Figueiredo ICR, Bernal R, Silva NN. Monitoramento de fatores de risco para doenças crônicas por entrevistas telefônicas. Rev Saude Publica 2005; 39(1):47-57.

18. Claro RM, Santos MAS, Oliveira TP, Pereira CA Szwarcwald CL, Malta DC. Consumo de alimentos não saudáveis relacionados a doenças crônicas não transmissíveis no Brasil: Pesquisa Nacional de Saúde, 2013. Epidemiol E Serviços Saúde 2015; 24(2):257-265.

19. Neves ACM, Moura EC, Gonzaga LAA, Martens IBG. Validação de indicadores do consumo de alimentos e bebidas obtidos por inquérito telefônico em Belém, Pará, Brasil. Cad Saude Publica 2010; 20(12):2379-88.

20. World Health Organization (WHO). Global Recommendations on Physical Activity for Health [Internet]. [acessado 2017 Set 15]. Disponível em: http://apps.who.int/iris/bitstream/handle/10665 /44399/9789241599979_eng.pdf;jsessionid=ED 6665028371354DE0B8F1E4BA968997? sequence $=1$

21. Shaikh RA, Siahpush M, Singh GK, Tibbits M. Socioeconomic Status, Smoking, Alcohol use, Physical Activity, and Dietary Behavior as Determinants of Obesity and Body Mass Index in the United States: Findings from the National Health Interview Survey. Int $J \mathrm{MCH}$ AIDS 2015; 4(1):22-34.

22. Cassidy S, Chau JY, Catt M, Bauman A, Trenell MI Low physical activity, high television viewing and poor sleep duration cluster in overweight and obese adults; a cross-sectional study of 398,984 participants from the UK Biobank. Int J Behav Nutr Phys Act 2017; 14(1):57-67.

23. Meneguci J, Teles Santos DA, Barboza Silva R, Gomes Santos R, Sasaki JE, Tribess S, Damião R, Virtuoso Júnior JS. Comportamento sedentário: conceito, implicações fisiológicas e os procedimentos de avaliação. Motricidade 2015; 11(1):160-174.

24. Rezende LFM, Lopes MR, Rey-López JP, Matsudo VKR, Luiz OC. Sedentary Behavior and Health Outcomes: An Overview of Systematic Reviews. PLoS One 2014; 9(8):e105620.

25. Chau J, Chey T, Burks-Young S, Engelen L, Bauman A. Trends in prevalence of leisure time physical activity and inactivity: results from Australian National Health Surveys 1989 to 2011. Aust N Z J Public Health 2017; 41(6):617-624.

26. Goularte Knuth A, Carvalho Malta D, Carvalho Dumith S, Azeredo Pereira C, Libânio Morais Neto O, Gomes Temporão J, Penna G, Hallal PC. Prática de atividade física e sedentarismo em brasileiros: resultados da Pesquisa Nacional por Amostra de Domicílios (PNAD) - 2008. Cien Saude Colet 2011; 16(9):36973705.
27. Mielke GI, Hallal PC, Rodrigues GBA, Szwarcwald CL, Santos FV, Malta DC. Prática de atividade física e hábito de assistir à televisão entre adultos no Brasil: Pesquisa Nacional de Saúde 2013. Epidemiol E Serviços Saúde 2015; 24(2):277-286.

28. Jashinsky J, Gay J, Hansen N, Muilenburg J. Differences in TV Viewing and Computer Game Playing's Relationships with Physical Activity and Eating Behaviors among Adolescents: An NHANES Study. Am J Health Educ 2017; 48(1):41-47.

29. Pearson N, Griffiths P, Biddle SJ, Johnston JP, McGeorge S, Haycraft E. Clustering and correlates of screen-time and eating behaviours among young adolescents. BMC Public Health 2017; 17(1):533-545.

30. Nooijen CFJ, Möller J, Forsell Y, Ekblom M, Galanti MR, Engström K. Do unfavourable alcohol, smoking, nutrition and physical activity predict sustained leisure time sedentary behaviour? A population-based cohort study. Prev Med 2017; 101:23-27.

31. Austys D, Palionis D, Valeviciene N, Stukas R. Consumption of sweets and adipose tissue distribution in adults with cardiovascular diseases Donatas Austys. Eur J Public Health 2017; 27(Supl. 3):187-228.

32. Trumbo PR, Rivers CR. Systematic review of the evidence for an association between sugar-sweetened beverage consumption and risk of obesity. Nutr Rev 2014; 72(9):566-574.

33. Nicholas JA, Siou GL, Lynch BM, Robson PJ, Friedenreich CM, Csizmadi I. Leisure-Time Physical Activity Does not Attenuate the Association Between Occupational Sedentary Behavior and Obesity: Results From Alberta's Tomorrow Project. J Phys Act Health 2015; 12(12):1589-600

34. Ekelund U, Steene-Johannessen J, Brown WJ, Fagerland MW, Owen N, Powell KE, Bauman A, Lee IM Lancet Physical Activity Series 2 Executive Committe; Lancet Sedentary Behaviour Working Group. Does physical activity attenuate, or even eliminate, the detrimental association of sitting time with mortality? A harmonised meta-analysis of data from more than 1 million men and women. Lancet 2016; 388(10051):1302-1310.

35. Maher CA, Mire E, Harrington DM, Staiano AE, Katzmarzyk PT. The independent and combined associations of physical activity and sedentary behavior with obesity in adults: NHANES 2003-06. Obesity 2013 21(12):E730-737.

36. Del Duca GF, Nahas MV, Silva DAS, Hallal PC, Malta DC, Peres MA. Physical activity indicators in adults from a state capital in the South of Brazil: a comparison between telephone and face-to-face surveys. Cad Saude Publica 2013; 29(10):2119-2129.

Artigo apresentado em 17/05/2018

Aprovado em 14/10/2018

Versão final apresentada em 16/10/2018 
